\title{
An aortic dissection treated with left main coronary artery stent implantation
}

\author{
Goksel Guz ${ }^{1}$, Ibrahim Altun², Bilal Karaayvaz ${ }^{3}$, Fatih Akin ${ }^{2}$, Mehmet Kocaaga $^{3}$, Sebahattin Atesal $^{1}$ \\ 1Department of Cardiology, Istanbul Medicana International Hospital, Istanbul, Turkey \\ ${ }^{2}$ Department of Cardiology, Faculty of Medicine, Mugla Sitki Kocman University, Mugla, Turkey \\ ${ }^{3}$ Department of Cardiology, Istanbul Medical Faculty, Istanbul University, Istanbul, Turkey
}

Postep Kardiol Inter 2014; 10, 4 (38): 314-316

DOI: $10.5114 /$ pwki.2014.46777

\begin{abstract}
A b s tract
Retrograde coronary aortic dissection is a rare but dangerous complication of coronary angioplasty. Mostly seen in right coronary artery procedures, especially in chronic total oclussion lesions, and rarely seen in left side procedures. This is a case report of an aortic dissection complicated by coronary angioplasty of the left circumflex artery. Immediate stenting of the left main coronary artery successfully sealed the entry point of dissection and stabilized the patient.
\end{abstract}

Key words: aorta, dissection, stenting.

\section{Case report}

A 57-year-old patient has admitted to our clinic with chest pain. He had hypertension and hyperlipidaemia. His exercise ECG testing was positive for ischaemia. His coronary angiography showed $80 \%$ stenosis in the left circumflex (LCX) artery and a bare metal stent (BMS) was implanted. In control angiography there was a notch in left main coronary artery (LMCA), but due to haemodynamic stability, no chest pain, and uncertainty we ignored the notch. Four hours after the stent implantation a sudden hypotension and swelling started and the patient was immediately taken to the cath lab. His coronary angiography showed a dissected segment proximal to the previously implanted stent. With contrast given to see the dissected LCX segment, spiral dissection went further from LMCA into left anterior descending (LAD). A floppy guidewire immediately sent for LAD and fortunately went through the dissection. After that a BMS stent was implanted from mid LMCA to proximal LAD. In control angiography a dissection was seen in LMCA under the stent with progression to the aorta, which caused opaque jamming (Figures 1, 2). Cardiovascular surgeons were informed. In a joint decision with cardiovascular surgeons, we planned ostial LMCA stenting to prevent the retrograde progression of the aortic dissection. A BMS was implanted in the LMCA ostium. After a little while blood pressure rose and the patient stabilised. The patient was taken to the cardiovascular surgery care unit for follow up. The anginal chest pains that had persisted in his first 12 h eventually stopped. His contrast-enhanced thoracic computed tomography confirmed the unprogressed dissection (Figure 3). After 2 days in control contrast-enhanced thoracic computed tomography the thrombus in dissected lumen suggested regration (Figure 4).

\section{Discussion}

Retrograde coronary aortic dissection is a rare but dangerous complication of coronary angioplasty. The aortocoronary dissection mostly originates from catheter-induced trauma and floppy induced trauma, balloon inflation, especially to the LMCA and RCA ostial lesions, forceful contrast injection, and harsh movement with devices (stents, etc.) in coronary arteries. Surgery is the choice of treatment in aortic dissections, but in these retrograde dissections ostial stenting is an alternative treatment [1-5].

In an article Shorrock et al. published 336 chronic total oclussion (CTO) percutaneous coronary intervention (PCI) cases from 2005 to 2012 in which they saw 6 retrograde aortic dissections that all arose from RCA. Four of these were treated with ostial stenting. In $107 \mathrm{PCl}$ cases

\section{Corresponding author:}

Ibrahim Altun Assist. Prof., Department of Cardiology, Faculty of Medicine, Mugla Sitki Kocman University, 48000 Mugla, Turkey, phone: +90 (252) 2115174, fax: +90 (252) 2111345, e-mail: ibrahim_altun@yahoo.com

Received: 14.06.2014, accepted: 9.09.2014. 


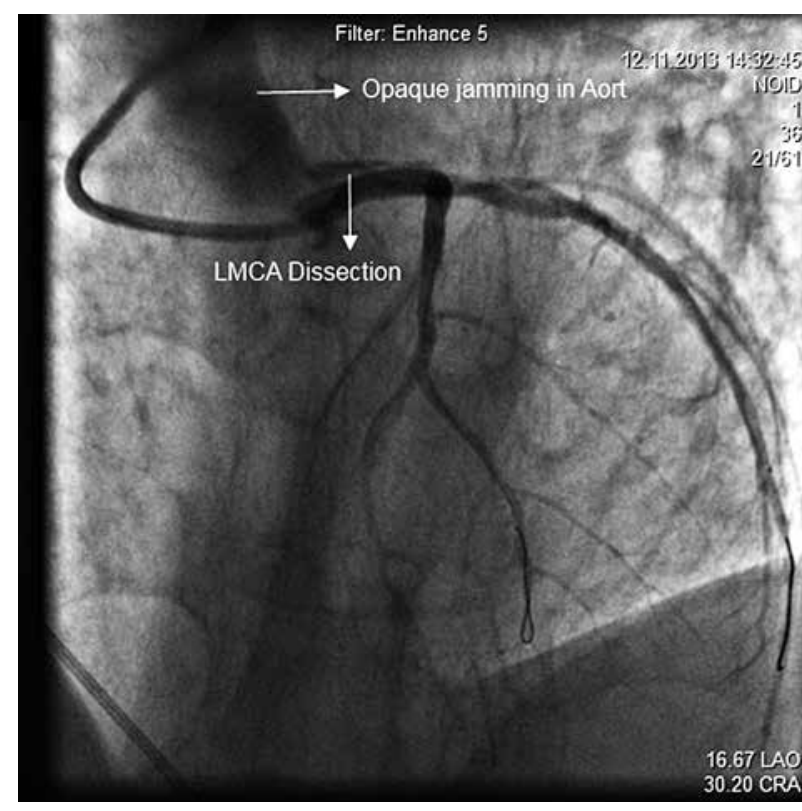

Figure 1. The LMCA dissection and opaque jamming in ascending aorta

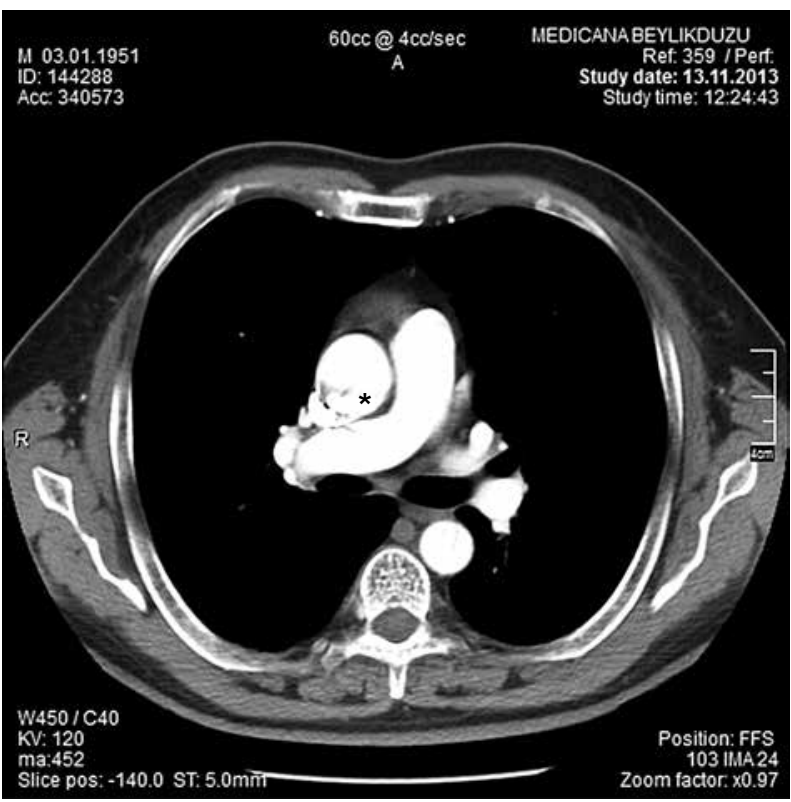

Figure 3. Contrast-enhanced thoracic computed tomography after $24 \mathrm{~h}$

${ }^{\star}$ Aortic dissection

in the literature, in which aortocoronary dissection occurs $78.4 \%$ were RCA, $49.5 \%$ were treated with stenting, and $29 \%$ with emergency coronary artery bypass grafting (CABG), and $21.5 \%$ conservatively. Of these patients 8 died, but none was from the stenting group [6].

When a coronary dissection is detected it is advised not to use contrast injection because giving contrast advances the dissection, which makes the situation even worse with every injection, as in our case. There are cases in the literature in which intravascular ultrasound

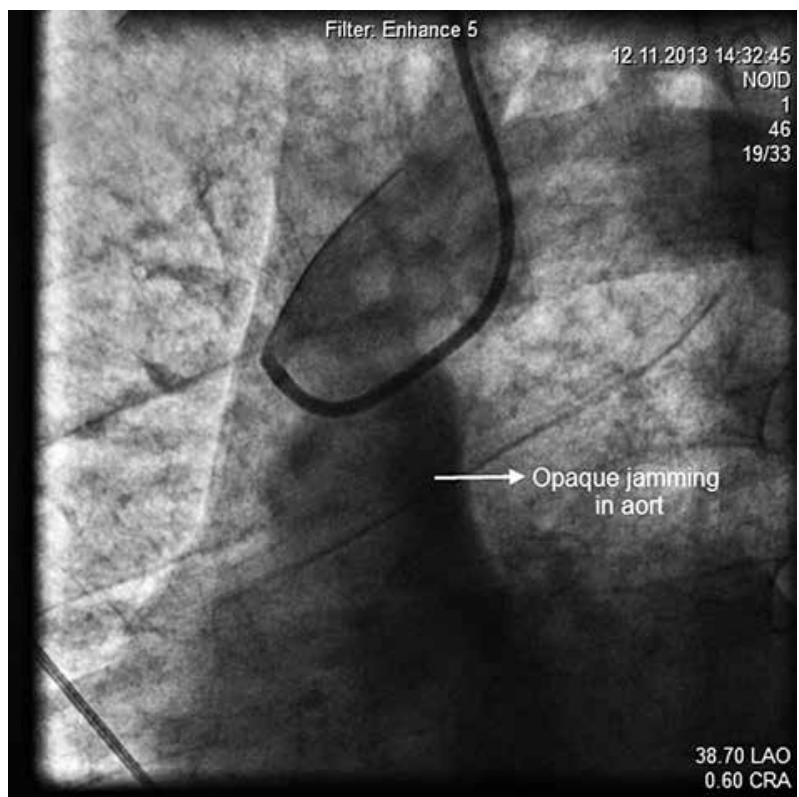

Figure 2. Opaque jamming while aorta root injection

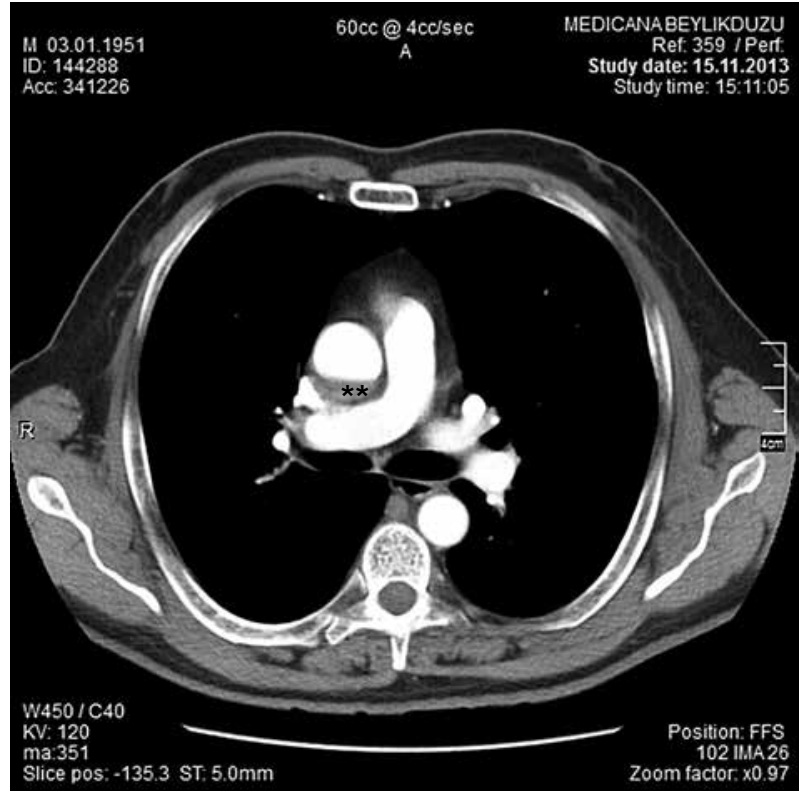

Figure 4. Contrast-enhanced thoracic computed tomography after $48 \mathrm{~h}$

**Thrombosed dissection

(IVUS)-guided stent implantation was successfully carried out without contrast use [7-9]. In our patient the dissection of aorta occurred during the angiography. Due to the patient's rapid deterioration, we had no time for IVUS, thus we were forced to use contrast to guide the stenting.

The dissection should be supervised, whether it is treated or not, with frequent controls because it can still persist or relapse in some cases, despite ostial stenting. 


\section{Conclusions}

Ostial stenting of the responsible artery is a fair alternative to emergency CABG and may be even better when the mortality and complicity of the surgery of acute dissection is taken into account. Further investigation is needed to determine the role of these treatment options.

\section{References}

1. Oda H, Hatada K, Sakai K, et al. Aortocoronary dissection resolved by coronary stenting guided by intracoronary ultrasound. Circulation 2004; 68: 389-91.

2. Carstensen S, Ward MR. latrogenic aortocoronary dissection: the case for immediate aortoostial stenting. Heart Lung Circulation 2008; 17: 325-9.

3. Lao EP, Nie SP, Ma CS. Immediate bail-out TAP-stenting for the treatment of iatrogenic aortocoronary dissection involving left main bifurcation. J Geriatr Cardiol 2013; 10: 202-4.

4. Fang HY, Wu CJ. Migrating type A intramural hematoma after repair of an anomalous right coronary artery aortocoronary dissection with a covered stent. Catheter Cardiovasc Intervent 2011; 78: 573-6.

5. Cheng CC, Tsao TP, Tzeng BH, et al. Stenting for coronary intervention-related dissection of the left main coronary artery with extension to the aortic root: a case report. South Med J 2008; 101: 1165-7.

6. Shorrock D, Michael TT, Patel V, et al. Frequency and outcomes of aortocoronary dissection during percutaneous coronary intervention of chronic total occlusions: a case series and systematic review of the literature. Catheter Cardiovasc Interv 2014; 84: 670-5.

7. Abdou SM, Wu CJ. Treatment of aortocoronary dissection complicating anomalous origin right coronary artery and chronic total intervention with intravascular ultrasound guided stenting. Catheter Cardiovasc Interv 2011; 78: 914-9.

8. Sakakura K, Wada H, Taniguchi Y, et al. Intravascular ultrasound-guided coronary stenting without contrast medium for the treatment of catheter-induced aortocoronary dissection. Cardiovasc Interv Therap 2013; 28: 71-5.

9. Kano N, Kawaguchi K, Funabiki J, et al. Intravascular ultrasound-guided percutaneous coronary intervention for total occlusion of the proximal left anterior descending artery caused by spontaneous coronary dissection in a young woman. Cardiovasc Interv Therap 2012; 27: 31-5. 